\section{Inhaled Plastic Cap from Firework: Report of Two Cases}

Brit.med. F., 1966, 2, 1052

\section{CASE 1}

A boy aged 8 years 10 months was admitted to Abergele Chest Hospital on 13 December 1965, having had a cough for two months, but it seemed that his cough became really troublesome after 5 November He was said to have lost weight and to have had periodic abdominal pain. There was a history of contact with two uncles who had suffered from tuberculosis, and because of this he had been given B.C.G. vaccination at birth.

On referral to the chest clinic on 3 December he was found to have slight finger-clubbing and dullness, with diminished breath sounds' at the right base. Chest $x$-ray examination showed a dense opacity due to collapse of the right lower and middle lobes. His tuberculin skin test was positive by the multiple-puncture method.

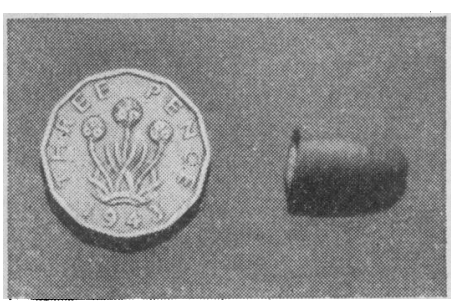

Fic. 1.- Case 1. Cap of firework coughed up. Because of the history of contact, clinical findings, and $x$-ray appearances, he was sent to hospital on 13 December.

On admission he looked well but had a very severe cough, which led the nursing staff to think he might have whooping-cough. This type of paroxysmal cough had been noticed in tuberculous children with enlarged glands,

and it was thought that this might be the cause. His temperature varied between 97 and $98.6^{\circ}$ F. (36.1 and $37^{\circ}$ C.). B.S.R. was $14 \mathrm{~mm}$. by the Westergren method in the first hour; $\mathrm{Hb} 80 \%$; W.B.C. $7,900 / \mathrm{cu}$. mm. (neutrophils $56 \%$, basophils $1 \%$, lymphocytes $35 \%$, monocytes $7 \%$ ). Sputum tests for acid-fast bacilli were negative on smear, and culture.

On 17 December he coughed out a blunt-nosed hollow piece of red plastic material $\frac{1}{2}$ in. (12.5 mm.) long and $\frac{1}{4}$ in. $(6.25 \mathrm{~mm}$.) across at its base (Fig. 1). This was the cap of a firework. He gave a history that on the night of 5 November he pulled this cap off a firework by holding the cap with his teeth and pulling on the base of the firework. He thought at the time that he had swallowed the cap and did not inform anyone.

On re-examination of the clinic films taken before admission to hospital the foreign body could with difficulty be seen lying at the level of the posterior end of the seventh right rib. Further films taken on 31 January 1966 showed some clearing of the collapse in the right lower and middle lobes. A bronchogram done on 20 January showed marked bronchiectasis in the middle lobe and

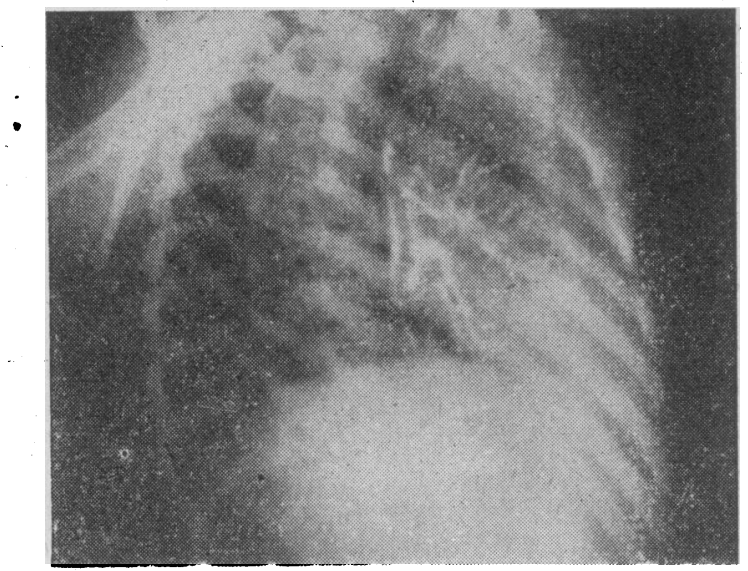

Fig. 2.-Case 2. Bronchogram showing complete block of left lower bronchus. in all segments of the lower lobe except the apical segment. By 9 February there had been some further clearing, but the lower lobe still remained collapsed in part.

At the time of discharge he still had slight finger-clubbing. His E.S.R. was $10 \mathrm{~mm}$. in the first hour. There were medium rales over the lower quarter of the right lung both anteriorly and posteriorly.

\section{CASE 2}

A similar incident occurred in a boy aged 9 who was admitted to a local general hospital on 29 September 1965 with a history of having had a choking attack after holding between his teeth the red plastic cap of a "banger." He was transferred to Abergele Chest Hospital for further investigation. Before admission he had developed a collapsed left lower lobe, and at bronchoscopy on 30 September there was much congestion and oedema in the left lower lobe bronchus, but the foreign body could not be seen. A bronchogram done on 4 October showed complete block of the left lower lobe bronchus by a thimble-like cap plugging the bronchus. The opaque medium used entered the cap, and this shows up well on the bronchogram (Fig. 2). A further bronchoscopy was done on the same day, but no recognizable rim or part of the foreign body could be seen. The bronchus was very oedematous. As it was certain, nevertheless, what the condition was due to, thoracotomy was performed. The plastic cap was easily palpated in the left lower lobe bronchus, where it was tightly impacted, and it was removed by bronchotomy.

The boy made a smooth recovery and was discharged 14 days later, chest $x$-ray examination having shown good re-expansion of the left lower lobe. At the time of writing his condition was excellent, he had no chest symptoms or abnormal physical signs in his chest, and his $x$-ray picture was quite normal.

\section{COMMENT}

Information supplied to the Ministry of Health by hospitals in England and Wales of patients receiving treatment for firework injuries between 17 October and 13 November 1965 showed that there was a total of 2,339 incidents. The majority arose from "bangers" ; in all there were 618 injuries from this type of firework. No specific mention is made of any accidents involving the inhalation of part of a firework, and a personal communication from the Home Office indicated that no reports had been received of this type of accident. On being contacted one of the firework manufacturers indicated that capping of "bangers" had been suggested several years ago as a safety measure. Some manufacturers, however, did not agree with this method of ensuring the safety of the product, but decided that it would be more satisfactory to sell "bangers" in flip-top cartons rather like a type of cigarette packet, and to insert the firework business-end downwards. The Home Office communication indicated also that other devices and packages are fitted with plastic caps which could possibly be inhaled. This being so, it behoves manufacturers requiring safety devices for their products to employ methods which avoid the use of small pieces of plastic which are likely to get into the mouths of children.

Because of the colour, the caps concerned in the cases described could not easily be seen on bronchoscopy, being indistinguishable from the inflamed mucous membrane and impossible to identify. Moreover, they are just the right size to block the lower lobe bronchus of boys aged 8 to 12 years. If they are lying apex downwards in the bronchus the upper rim is embedded in oedematous mucosa. This not only causes impaction but makes the rim impossible to see.

I am indebted to Dr. Glyn Jones, who sent in the first patient, and to Mr. Ivor Lewis for permission to record the second patient, upon whom he operated.

Abergele Chest Hospital, J. B. MORRISON, M.D., B.SC. 